\title{
Nano-porous Silicon Microcavity Sensors for Determination of Organic Fuel Mixtures
}

\author{
Van Hoi Pham ${ }^{1 *}$, Huy Bui ${ }^{1}$, Le Ha Hoang ${ }^{2}$, Thuy Van Nguyen ${ }^{1}$, The Anh Nguyen', \\ Thanh Son Pham ${ }^{1}$, and Quang Minh Ngo ${ }^{1}$ \\ ${ }^{1}$ Institute of Materials Science, VAST, 18 Hoang Quoc Viet Rd., Cau giay Dist., Hanoi, Vietnam \\ ${ }^{2}$ Department of Electronics and Telecom, College of Sciences, Hue University, 77 Nguyen Hue Str., \\ Hue, Vietnam
}

(Received July 1, 2013 : revised September 3, 2013 : accepted September 3, 2013)

\begin{abstract}
We present the preparation and characteristics of liquid-phase sensors based on nano-porous silicon multilayer structures for determination of organic content in gasoline. The principle of the sensor is a determination of the cavity-resonant wavelength shift caused by refractive index change of the nano-porous silicon multilayer cavity due to the interaction with liquids. We use the transfer matrix method (TMM) for the design and prediction of characteristics of microcavity sensors based on nano-porous silicon multilayer structures. The preparation process of the nano-porous silicon microcavity is based on electrochemical etching of single-crystal silicon substrates, which can exactly control the porosity and thickness of the porous silicon layers. The basic characteristics of sensors obtained by experimental measurements of the different liquids with known refractive indices are in good agreement with simulation calculations. The reversibility of liquid-phase sensors is confirmed by fast complete evaporation of organic solvents using a low vacuum pump. The nano-porous silicon microcavity sensors can be used to determine different kinds of organic fuel mixtures such as bio-fuel (E5), A92 added ethanol and methanol of different concentrations up to $15 \%$.
\end{abstract}

Keywords : Microcavity devices, Optical sensors, Multilayers, Electrochemical etching

OCIS codes : (140.3948) Microcavity devices; (130.6010) Sensors; (230.4170) Multilayers

\section{INTRODUCTION}

Recently, porous silicon (PS) has generated a great interest for use in photonic structures and optical devices. Several studies of PS were devoted to understanding the formation mechanisms of pores and porous multilayer structures and the relationship of PS with its physical and chemical properties that can be altered easily just by controlling the current density and electrolyte solution in the electrochemical etching process [1-3]. The one-dimensional porous silicon photonic crystal (1D-PSPC) devices such as optical filters and microcavities in the PS technology have been achieved by multilayer structure formation with periodic refractive index sequence [4]. The periodic sequence was obtained by controlling the current density and etching time in a periodic way while the others electrochemical parameters were fixed [5]. The PS photonic crystal structures have large potential for use in the optical sensor technology, especially in the gas and liquid-phase sensors [6-9]. The specifications of these optical devices are the porous structure of constituent layers that showed high specific surface area for interaction with ambient gas or liquid. Then the nanoporous structural features have allowed fabrication of high sensitivity gas, chemical and/or biosensors [10-12]. In the literature on the nano-PS photonic sensors, most papers show PS distributed feedback (DFB) structures with large reflectance spectra that limited the accuracy of the measurement. In addition, the role of porosity and its contrast with the multilayer structures, which strongly depends on preparation process, still has not been discussed.

In this paper, we present research results on preparation of the liquid-phase photonic sensors based on nano-PS microcavity structures and the influences of the configuration and porosity of multilayers on the sensitivity and accuracy of measurements using simulation and experiments. We show the fabrication process and characteristics of liquid-

\footnotetext{
*Corresponding author: hoipv@ims.vast.ac.vn

Color versions of one or more of the figures in this paper are available online.
} 
phase sensors based on nano-PS microcavity using an electrochemical etching method. Simulation study is based on the transfer matrix method (TMM), which developed our previous results in ref. [13] for prediction of porosity of multilayers. The sensor response is given by the shift of the resonant wavelength of the microcavity when the sensors are immersed in liquids. A change of sensor reflectance spectra is temporary, that characteristic is useful for reversible optical sensing. The nano-porous silicon microcavity sensors can be used to investigate different kinds of gasoline such as bio-fuel (E5), A92 added ethanol and methanol with concentrations of $5 \%-15 \%$.

\section{DESIGN AND PREPARATION OF NANO-POROUS SILICON MICROCAVITY}

For design of nano-porous silicon microcavity (PSM), we use the TMM for calculation of the parameters of multilayer structures. The microcavity structure was designed by using the $(\mathrm{HL})^{n} \mathrm{LL}(\mathrm{HL})^{m}$ sequence, where $\mathrm{H}$ and L labels correspond to high and low refractive index layers, respectively, and $n$ (and/or $m$ ) is a quantity of HL pairs. In the sequence above, the HL pair is the unitary cell of photonic crystal (PC) structure and the LL pair is the microcavity structure (defect layer) localized between two Bragg mirrors created by PC layers [13]. The spectral region of the optical response for the 1D-PSPC device was projected by using Bragg's relation, where the thickness of the defect layer was equal to $\lambda / 2$ or $\lambda$, as follows:

$$
\lambda / 4=\mathrm{n}_{\mathrm{H}} \cdot \mathrm{d}_{\mathrm{H}}=\mathrm{n}_{\mathrm{L}} \cdot \mathrm{d}_{\mathrm{L}}
$$

In this equation $\lambda$ is the wavelength corresponding to the center of the photonic band gap (PBG) region; $n_{H}$ and $n_{L}$ $\left(\mathrm{d}_{\mathrm{H}}\right.$ and $\left.\mathrm{d}_{\mathrm{L}}\right)$ are the refractive indices (thicknesses) of the $\mathrm{H}$ and $\mathrm{L}$ layers, respectively.

Porous silicon layers with different refractive indices were formed by electrochemical etching of a highly doped p-type silicon wafer (resistivity is of $0.01 \div 0.1 \Omega \mathrm{cm}$ ) in an aqueous HF: ethanol electrolyte, because the p-type silicon substrates yield the most favorable porous silicon morphology. The electrolyte with concentration of $16 \% \mathrm{HF}$ and ethanol of ratio $1: 2$ is chosen because of the large porosity variation obtained by varying the electrical current. The electrochemical etching process was controlled by computer program using Galvanostat equipment (Autolab PGSTAT 30), so precise control over electrical current density and etching time was achieved. The result is a good control of the refractive index and thickness over the individual layers forming the multilayer.

Figure 1 shows the calculation result with a numerical simulation and the experimental measured reflective spectrum of the microcavity based on $(\mathrm{HL})^{4.5} \mathrm{LL}(\mathrm{HL})^{5}$ nano-porous silicon multilayer structure (4.5 means four and one half pairs of HL). The nano-porous silicon microcavity structure was obtained from anodization current densities of $15 \mathrm{~mA} / \mathrm{cm}^{2}$ and $50 \mathrm{~mA} / \mathrm{cm}^{2}$ and with etching times of $2.86 \mathrm{sec}$ and $4.76 \mathrm{sec}$ for high and low refractive index layers, respectively. The feature of the microcavity structures in the air characterized by a single resonance peak at wavelength of 506 $\mathrm{nm}$ in a transmittivity stopband in the wavelength range of $436 \mathrm{~nm}-600 \mathrm{~nm}$. The imperfection of interfaces between high and low refractive index layers created by the electrochemical etching caused the deviation of the simulation from the experimental reflective spectra. In general, the measured reflective spectrum of the microcavity is smaller than the calculated one.

Figure 2 shows the image of a cross-section of the nano-porous silicon multilayer microcavity (Fig. 2(a)) and

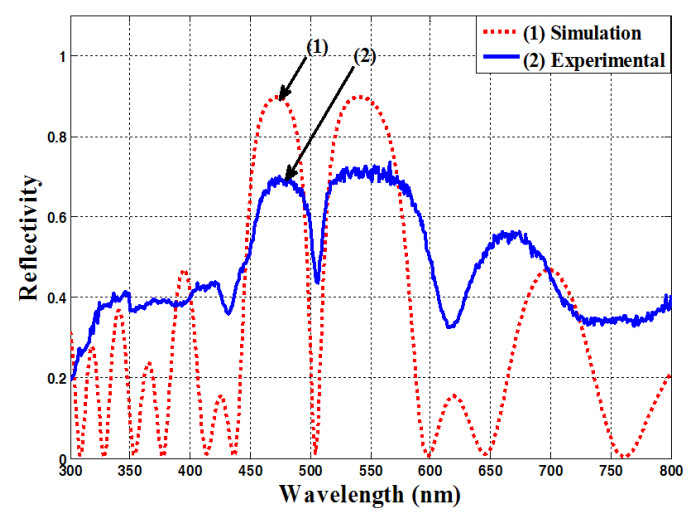

FIG. 1. Reflective spectra of microcavity device based on the nano-porous silicon multilayer structures. The line 1 is the numerical simulated spectrum (neglecting absorption and scattering) and the line 2 is the experimental measured one.
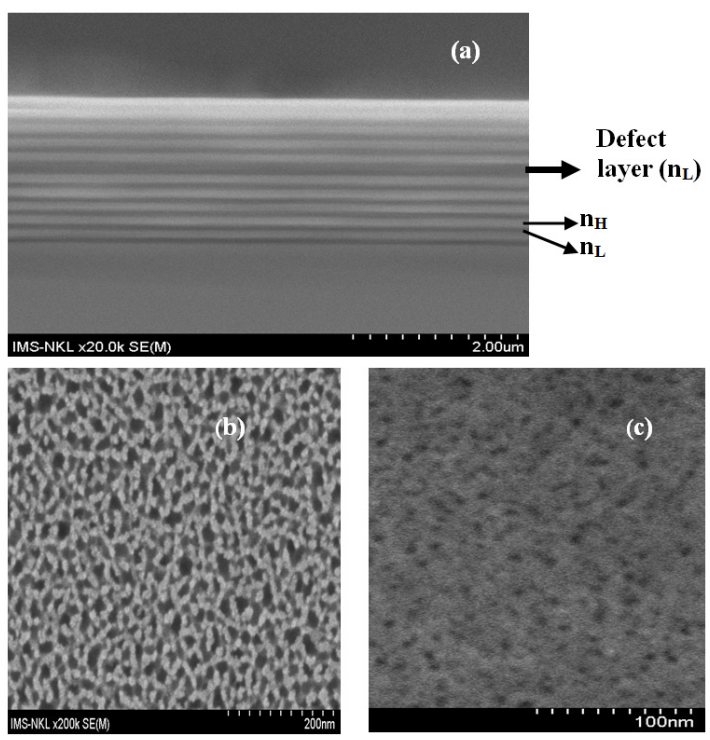

FIG. 2. (a) SEM cross-section view of nano-PS microcavity structure; (b) and (c) surface plan views of high and low reflective index nano-PS layers, respectively. 
the surface plan views of the nano-porous silicon layers with high (Fig. 2(b)) and low refractive index (Fig. 2(c)), captured by ultrahigh resolution field emission scanning electron microscopy (FE-SEM) S-4800. The silicon pores have a size of $20-25 \mathrm{~nm}$. For characterization of the nanoporous silicon microcavity, we used the ultraviolet-visiblenear infrared (UV-VIS-NIR) spectrophotometer (Varian Cary 5000) for measurement of reflective spectra of the samples. For prevention of aging in the porous silicon layers, we thermally annealed the samples at $200^{\circ} \mathrm{C}$ in nitrogen ambient for a time of 60 minutes. After annealing, the resonant wavelength peak shifted 1.0-1.5 nm into the short-wavelength zone from the increasing pore density of the layers.

\section{CHARACTERISTICS OF LIQUID-PHASE PHOTONIC SENSORS}

The basic characteristics of the porous silicon microcavity (PSM) and the resonant wavelength shift $(\Delta \lambda)$ caused by the ambient refractive index (n), were built experimentally by using a series of liquids with known refractive indices. The effective refractive index of the nano-porous silicon layer immersed into organic solvent would be increased due to the substitution of air with liquid in the pores and consequently the optical thickness of the layer is increased. As a result, the resonant wavelength shift would be dependent upon the refractive index value of the organic solvent. Table 1 presents a series of organic solvent such as methanol $99.5 \%$, ethanol $99.7 \%$, isopropanol $99.7 \%$ and methylene chloride $99.5 \%$ (product of NHTC-China) with their refractive indices and the resonant wavelengths of the sensors, when they are dipped into the corresponding organic solvent for some minutes.

Sensitivity $(\Delta \lambda \Delta n)$ is one of the most important parameters to evaluate the performance of the sensors. Using the experimental data in Table 1, we calculate the sensor sensitivity of about $200 \mathrm{~nm} / \mathrm{RIU}$. The Spectrophotometer Varian Cary 5000 is able to detect a wavelength shift of $0.1 \mathrm{~nm}$, corresponding to the minimum detectable refractive index change in the porous silicon layer of less than $10^{-3}$. Experiment shows that after complete evaporation of organic solvent, the reflectance spectra of the sensors return to their original

TABLE 1. Various organic solvents with known refractive index and resonant wavelengths of sensors based on porous silicon microcavity dipped in corresponding solvent

\begin{tabular}{c|c|c}
\hline \hline Organic Solvent & $\begin{array}{c}\text { Refractive } \\
\text { index }\end{array}$ & $\begin{array}{c}\text { Resonant } \\
\text { wavelength (nm) }\end{array}$ \\
\hline Air & 1.0003 & 504.75 \\
Methanol (99.5\%) & 1.3280 & 572.05 \\
Ethanol (99.7\%) & 1.3614 & 579.00 \\
Isopropanol (99.7\%) & 1.3776 & 583.17 \\
Methylene chloride (99.5\%) & 1.4242 & 592.85 \\
\hline
\end{tabular}

waveform positions (as in the air). In our case the evaporation of organic solvents in open air at room temperature was carried out for 40-50 minutes, but this process can occur in 20 seconds when the samples are in a vacuum chamber with $10^{-1}$ torr. That means, the change of sensor reflectance spectra are temporary, and it is useful for reversible optical sensing.

An important parameter of the microcavity sensor is that the change of the refractive index of the porous layer depends on the refractive index of the liquid as well as on the porosity of the porous layer. In Bruggeman effective medium approximation, the relation between effective refractive index of the pore layers $\left(n_{P S i}\right)$, silicon refractive index $\left(n_{S i}=3.5\right)$, void refractive index $\left(n_{\text {void }}=1\right)$ and porosity $(P)$ is presented by the following equation:

$$
(1-P) \frac{n_{S i}^{2}-n_{P S i}^{2}}{n_{S i}^{2}+2 n_{P S i}^{2}}+P \frac{n_{\text {void }}^{2}-n_{P S i}^{2}}{n_{\text {void }}^{2}+2 n_{P S i}^{2}}=0
$$

Based on this relation we determined the porosity $(P)$ of the pore layer and the refractive index of the pore layer $\left(n_{P S i}\right)$ dipped in liquid with known refractive index. The simulation calculations for basic characteristics of the sensor consist of the following steps:

1. Determination of the refractive index of the defect layer based on the experimental resonant wavelength and the pore layer thickness from the scanning electron microscopy (SEM) image.

2. Determination of layer porosity in air using the relation (2). When the refractive indices of the pore layers in air changed from 2.739 to 1.323 , the porosity of the layer changed from 30 to 80 by calculation.

3. Determination of effective refractive index of the layer when the voids were filled by liquid with known refractive index using the relation (2).

4. Determination of resonant wavelength of the sensor dipped into liquid using the simulation reflectance spectra.

Simulation shows that the contrast of the porosities (i.e. refractive indices) of the layers strongly influences the wavelength shift (i.e. on the sensitivity) of the microcavity. The contrast of the porosities would be high when the change of current density was large in the electrochemical etching process. However, the experiment shows that the imperfection of the interfaces of layers increased with the large change of current densities. In our work, when the porosity contrast of layers is more than 40 , the reflective spectra of the device were deformed in the reflection intensity and the line-width of the transmittance zone.

The curves from $\mathrm{C} 1$ to $\mathrm{C} 4$ in Fig. 3 present the fitting process of sensor basic characteristics by simulations with that by experiment (curve E). The fitting showed that the porosity contract between two layers affects the sensor sensitivity $(\Delta \lambda / \Delta \mathrm{n})$. Consequently, the matching process 


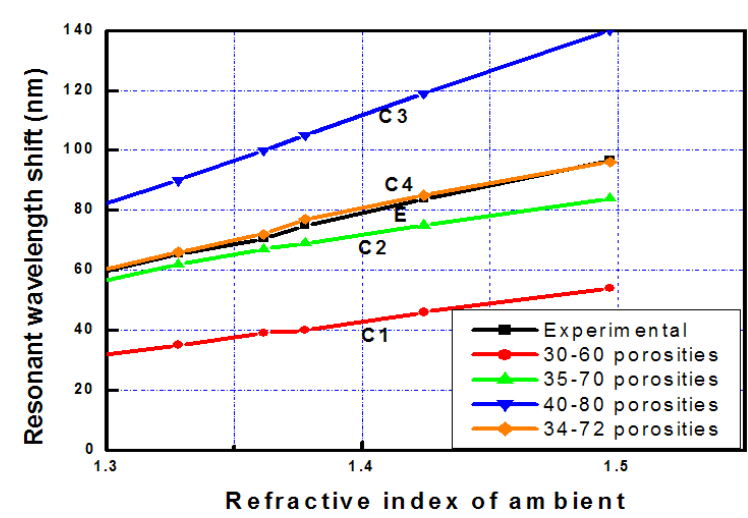

FIG. 3. The basic characteristics of liquid-phase sensor prepared from porous silicon microcavity obtained in experiment (curve E), and by simulation calculations (curves C1, C2, C3, C4) with various pairs of porosities.

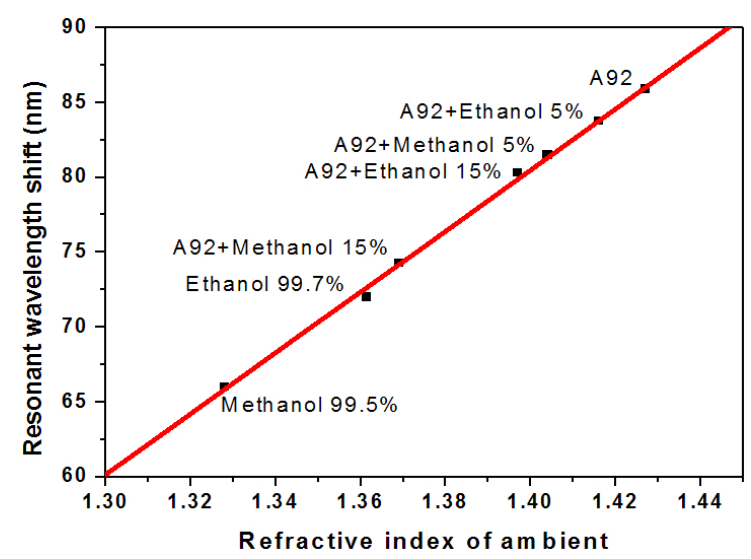

FIG. 4. Response characteristics of the sensor wavelength shift for mixture of methanol and ethanol in different concentrations and commercial gasoline A92.

found suitable porosity of $34 \%$ and $72 \%$ of low and high porosity layers of the prepared sensor, respectively.

The microcavity-based sensors have been applied to determination of different solutions of ethanol and methanol in the commercial gasoline A92. Figure 4 shows the measured results of the resonant wavelength shift of the microcavity sensor immersed into gasoline A92 with different concentrations of ethanol and methanol. In the case of a mixture of ethanol/A92, a resonant wavelength shift is $3.6 \mathrm{~nm}$, when ethanol concentration changed in the range from $5 \%$ to $15 \%$ in the gasoline. With the sensitivity of the sensor as described above, the minimum determination of ethanol concentration change in the gasoline is about $0.4 \%$. In the case of methanol/A92, wavelength shifts are $7.2 \mathrm{~nm}$ between the $5 \%$ and $15 \%$ methanol mixtures,. From these experimental data, we suppose that the enhanced sensor can distinguish change of about $0.2 \%$ in concentration of methanol in the gasoline.

\section{CONCLUSION}

In conclusion, we successfully fabricated a nano-porous silicon microcavity with resonant wavelength in the visible region and used them as liquid-phase photonic sensors. We built the basic characteristics of sensors by simulation calculations and by experiment based on a series of organic solvents with known refractive indices. Enhanced sensor sensitivity of $200 \mathrm{~nm} / \mathrm{RIU}$ can detect a minimum refractive index change of about $10^{-3}$. We used these sensors for determination of ethanol and methanol concentration from $5 \%$ to $15 \%$ in the commercial gasoline A92. Based on the experimental data we suppose that the enhanced sensors can distinguish a small concentration of methanol and ethanol in gasoline. This sensor, to our knowledge, is suitable to detect the very small content of methanol that damages the resin details in cars and motorbikes.

\section{ACKNOWLEDGMENT}

This work financially supported by National Foundation for Science and Technology Development (NAFOSTED) of Vietnam under Grant No.103.06.84.09. This work had been using the apparatus of National Key Laboratory for Electronic Materials and Devices in Institute of Materials Science.

\section{REFERENCES}

1. L. T. Canham, "Si quantum wire arrays fabrication by electrochemical and chemical dissolution of wafer," Appl. Phys. Lett. 57, 1046-1048 (1990).

2. H. J. Woo, G. D. Kim, H. W. Choi, and J. K. Kim, "Characterization of $\mathrm{SiC}$ nanostructures in crystalline and porous silicon formed by ion beam synthesis," J. Korean Phys. Soc. 56, 2063-2067 (2010).

3. H. Bui, V. H. Pham, H. K. Phan, T. V. Nguyen, and T. C. Do, "Porous silicon as a promising material for photonics," Int. J. Nanotechnol. 8, 360-370 (2011).

4. V. H. Pham, T. C. Do, H. Bui, and T. V. Nguyen, "Siliconrich silicon oxide thin films fabricated by electro-chemical method," in Optoelectronics-Materials and Techniques, P. Predeep, ed. (Publisher InTech Rijeka, Croatia, 2011), Chapter 2.

5. J. Gao, T. Gao, and M. J. Sailor, "Porous silicon vapor ensor based on laser interferometry," Appl. Phys. Lett. 77, 901-903 (2000).

6. Y. Shang, X. Wang, E. Xu, C. Tong, and J. Wu, "Optical ammonia gas sensor based on a porous silicon rugate filter coated with polymer-supported dye," Anal. Chim. Acta 685, 58-64 (2011).

7. M. S. Salem, M. J. Sailor, F. A. Harraz, T. Sakka, and Y. H. Ogata, "Sensing of chemical vapor using a porous multilayer prepares from lightly doped silicon," Phys. Stat. Sol. (c) 4, 2073-2077 (2007).

8. Y. Y. Kim, H. J. Kim, and K. W. Lee, "The potential for 
alcohol-containing gas sensor based on DFB porous silicon," J. Korean Phys. Soc. 55, 415-418 (2009).

9. L. D. Stefano, K. Malecki, F. G. Della Corte, L. Moretti, I. Rea, L. Rotiroti, and I. Rendina, "A microsystem based on porous Silicon-Gallss anodic bonding for gas and liquid potical sensing," Sensors 6, 680-687 (2006).

10. V. Mulloni and L. Pavesi, "Porous silicon microcavities as optical chemical sensors,” Appl. Phys. Lett. 76, 2523-2525 (2000).

11. W. Theiß, "Optical properties of porous silicon," Surf. Sci. Report 29, 91-93 (1997).
12. P. N. Patel, V. Mishra, and A. K. Panchal, "Theoretical and experimental study of nanoporous silicon photonic microcavity optical sensor devices," Adv. Nat. Sci.: Nanosci. Nanotechnol. 3, 035016 (2012).

13. T. C. Do, H. Bui, T. V. Nguyen, T. A. Nguyen, T. H. Nguyen, and V. H. Pham, "A microcavity based on a porous silicon multilayer," Adv. Nat. Sci.: Nanosci. Nanotechnol. 2, 035001 (2011). 